\title{
Flow-through stable isotope probing (Flow-SIP) minimizes cross-feeding in complex microbial communities
}

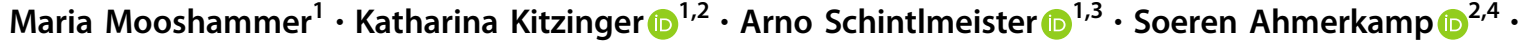 \\ Jeppe Lund Nielsen $\mathbb{1}^{5} \cdot$ Per Halkjær Nielsen $\mathbb{1}^{5} \cdot$ Michael Wagner $\mathbb{D}^{1,3,5}$
}

Received: 26 April 2020 / Revised: 3 August 2020 / Accepted: 24 August 2020 / Published online: 2 September 2020

(c) The Author(s) 2020. This article is published with open access

\begin{abstract}
Stable isotope probing (SIP) is a key tool for identifying the microorganisms catalyzing the turnover of specific substrates in the environment and to quantify their relative contributions to biogeochemical processes. However, SIP-based studies are subject to the uncertainties posed by cross-feeding, where microorganisms release isotopically labeled products, which are then used by other microorganisms, instead of incorporating the added tracer directly. Here, we introduce a SIP approach that has the potential to strongly reduce cross-feeding in complex microbial communities. In this approach, the microbial cells are exposed on a membrane filter to a continuous flow of medium containing isotopically labeled substrate. Thereby, metabolites and degradation products are constantly removed, preventing consumption of these secondary substrates. A nanoSIMS-based proof-of-concept experiment using nitrifiers in activated sludge and ${ }^{13} \mathrm{C}$-bicarbonate as an activity tracer showed that Flow-SIP significantly reduces cross-feeding and thus allows distinguishing primary consumers from other members of microbial food webs.
\end{abstract}

These authors contributed equally: Maria Mooshammer, Katharina Kitzinger

Supplementary information The online version of this article (https:// doi.org/10.1038/s41396-020-00761-5) contains supplementary material, which is available to authorized users.

Katharina Kitzinger

kkitzing@mpi-bremen.de

$\triangle$ Michael Wagner

michael.wagner@univie.ac.at

1 Division of Microbial Ecology, Centre for Microbiology and Environmental Systems Science, University of Vienna, Vienna, Austria

2 Max Planck Institute for Marine Microbiology, Bremen, Germany

3 Large-Instrument Facility for Environmental and Isotope Mass Spectrometry, Centre for Microbiology and Environmental Systems Science, University of Vienna, Vienna, Austria

4 MARUM-Center for Marine Environmental Sciences \& Department of Geosciences, University of Bremen, Bremen, Germany

5 Department of Chemistry and Bioscience, Aalborg University, Aalborg, Denmark
Stable isotope probing (SIP) is widely applied to link specific microbial populations to metabolic processes in the environment and has greatly advanced our understanding of the role of microorganisms in biogeochemical cycling. SIP relies on tracing the incorporation of specific isotopically labeled substrates (e.g., ${ }^{13} \mathrm{C},{ }^{15} \mathrm{~N},{ }^{18} \mathrm{O},{ }^{2} \mathrm{H}$ ) into cellular biomarkers or bulk cellular biomass [e.g., 1-4]. SIP is considered a robust technique to identify microbial populations that assimilate a labeled substrate of interest in complex environmental communities. However, crossfeeding can occur when isotopically labeled metabolites are released from a primary consumer and then used by other microorganisms, which subsequently also become isotopically labeled. Likewise, when ${ }^{13} \mathrm{C}$-bicarbonate and unlabeled substrate are supplied to assess the activity of specific chemolithoautotrophs [e.g., 5-7], undesired ${ }^{13} \mathrm{C}$-incorporation can occur due to cross-feeding between chemolithoautotrophs whose activity depends on each other, for example in nitrifiers, where ammonia oxidizers provide nitrite oxidizers with their substrate, nitrite. The uncertainties associated with cross-feeding in SIP studies increase as the incubation time of microbial communities increases. While this phenomenon can be used to study microbial interactions and trophic networks [8-11], crossfeeding can lead to erroneous identification of organisms 
that are not directly responsible for the process of interest, but are rather connected to primary consumers via a microbial food web [2, 10, 12, 13].

We developed an approach that significantly reduces the effect of cross-feeding in SIP studies. For this purpose, a thin layer of microbial cells is placed on a membrane filter, and isotopically labeled substrate is supplied at a fixed concentration by continuous flow, which constantly removes released metabolites and degradation products of primary substrate consumers. While previous SIP studies have employed a continuous flow of medium or substrate [e.g., 6, 14-16], in these studies, cross-feeding still occurred, as large amounts of biomass were placed in a 3D space, which allowed for the exchange of metabolites. Here, we present a proof-of-concept experiment with a nitrifying activated sludge microbial community, which converts ammonia to nitrite by the activity of ammonia-oxidizing bacteria (AOB), and subsequently oxidizes nitrite to nitrate by nitrite-oxidizing bacteria (NOB). In our experiments, the carbon source for both groups of autotrophic nitrifiers (the sludge contained no comammox bacteria [17, 18]) was isotopically labeled inorganic carbon $\left({ }^{13} \mathrm{C}-\mathrm{NaHCO}_{3}\right)$ and, as the sole electron donor, unlabeled ammonium was provided.

In the flow-through approach, $\mathrm{AOB}$, but not NOB, should be ${ }^{13} \mathrm{C}$-labeled because the substrate for NOB (nitrite), produced by $\mathrm{AOB}$ is continuously removed and thus the NOB should remain metabolically inactive (Fig. 1). In addition to a regular batch incubation, we included a control incubation, where the flow-through was recirculated

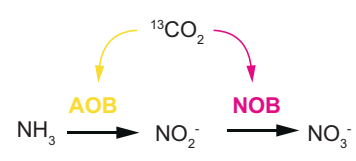

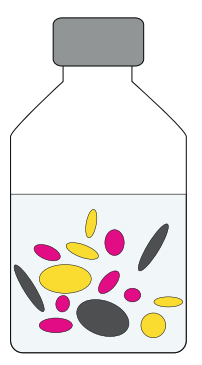

Batch

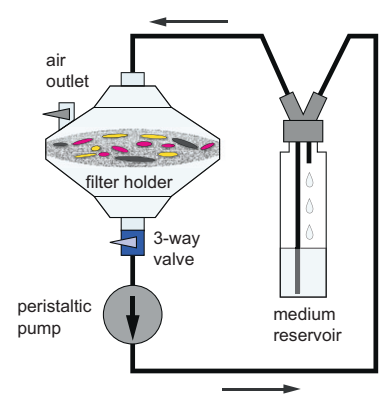

Recirculated to determine the impact of the experimental setup (continuous medium flow and retainment of biomass on a membrane filter) in Flow-SIP on the activity of the bacterial cells (in particular on the NOB as their autotrophic activity is used as a read out for cross-feeding in our experiments) in comparison to the batch experiment. Cross-feeding is expected to occur in both recirculated and batch control incubations, where nitrite is not removed and thus both AOB and NOB conserve energy to fix ${ }^{13} \mathrm{C}-\mathrm{CO}_{2}$. After the experiments, fluorescence in situ hybridization (FISH) with rRNA-targeted oligonucleotide probes was used to identify $\mathrm{AOB}$ and NOB and combined with nanoscale secondary ion mass spectrometry (nanoSIMS) to quantify ${ }^{13} \mathrm{C}$-assimilation at the single-cell level for all setups.

For these experiments, activated sludge from a Danish municipal wastewater treatment plant was initially treated by sonication to disrupt large flocs. Cells were then either placed on a membrane filter for flow-through incubation and the recirculated control experiment, or incubated in a conventional batch experiment. All experiments were set up using the same amount of biomass, and the ratio of biomass to medium volume was the same in batch and recirculated control experiments. Incubations were done using mineral medium containing $250 \mu \mathrm{M} \quad \mathrm{NH}_{4} \mathrm{Cl}$ and $2 \mathrm{mM}$ ${ }^{13} \mathrm{C}-\mathrm{NaHCO}_{3}$ for $24 \mathrm{~h}$. Medium flow was maintained at a rate of $26 \mathrm{ml} \mathrm{h}^{-1}$. We did not select a higher flow rate in order to avoid excessive stress by the medium flow on the microbial cells and to minimize the required amounts of media containing isotopically labeled bicarbonate. Furthermore, modeling nitrite advection and diffusion at different

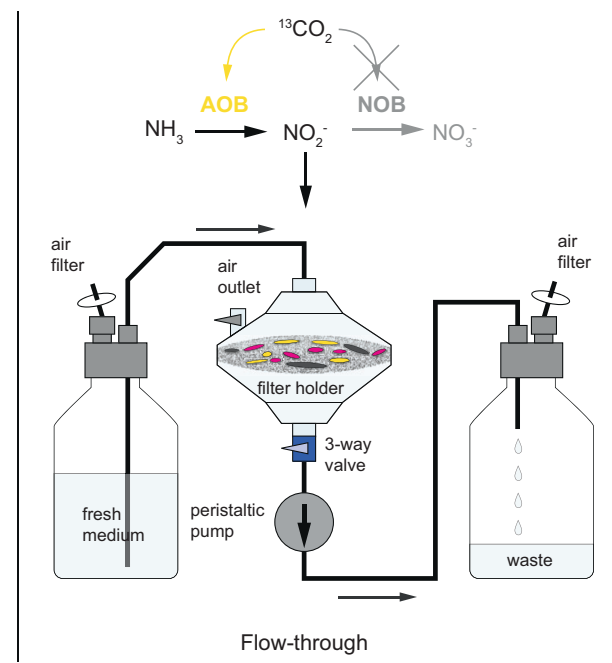

batch and recirculated approaches, where NOB consume nitrite produced via ammonia oxidation by AOB and thus both AOB and NOB incorporate ${ }^{13} \mathrm{C}-\mathrm{CO}_{2}$. In the flow-through approach, only $\mathrm{AOB}$ are expected to be ${ }^{13} \mathrm{C}$-labeled, as cross-feeding should be eliminated by the continuous removal of nitrite. Other, non-nitrifier cells are indicated in gray.
Fig. 1 Schematic representation of the experimental setup: (left) batch, (center) recirculated, and (right) flow-through incubation. In all incubations, the carbon source for both autotrophic nitrifiers (AOB, yellow and $\mathrm{NOB}$, magenta) was isotopically labeled (i.e., $\mathrm{CO}_{2}$ as ${ }^{13} \mathrm{C}-\mathrm{NaHCO}_{3}$ ) and ammonia was provided as the only external energy source (as $\mathrm{NH}_{4} \mathrm{Cl}$ ). Cross-feeding is expected to occur in the 
flow rates showed that, for example, a tenfold higher flow rate would only marginally reduce nitrite concentrations surrounding the AOB colonies (Fig. S1). In contrast, in a purely diffusive system without continuous flow, our model showed that nitrite would accumulate to significantly higher concentrations around single AOB colonies (Fig. S2). For example, after $24 \mathrm{~h}, \sim 23 \mu \mathrm{M}$ nitrite would accumulate at a distance of $0-100 \mu \mathrm{m}$ (with no significant decrease over distance) around an AOB colony of 50 cells, which is 230to 9200 -fold higher (depending on the distance to the colony) than modeled nitrite concentrations at the flow rate used in our experiments. Most inorganic metabolites that can directly be taken up into cells behave similar as nitrite in a diffusive system, i.e., they have a similar diffusion coefficient. Larger molecules tend to have an even smaller diffusion coefficient, which would lead to slower diffusion, and thus even more efficient metabolite removal when a medium flow is applied.

We monitored nitrification activity via concentration measurements of ammonium, nitrite and nitrate (Fig. S3) and conducted nanoSIMS analyses (Fig. 2) for two successive experiments using sludge collected from the same treatment plant on different days as replication of experimental results (referred to as E1 and E2). Additionally, we confirmed the reproducibility of the method with two further experiments, where nitrification activity was followed (Fig. S4). Details on the experimental setup are given in Fig. 1 and the Supplementary Text.

In recirculated and batch control incubations, the consumption of ammonium, production of nitrite and nitrate (Fig. S3), and single cell ${ }^{13} \mathrm{C}$-incorporation (Fig. 2) indicated that both $\mathrm{AOB}$ and $\mathrm{NOB}$ were active. However, nitrification activity (i.e., nitrite and nitrate production) in recirculated incubations were reduced by $57 \%$ (E1) and 83\% (E2) compared to batch incubations (Fig. S3). The reduced ammonia oxidation activity in the recirculated incubations compared to the batch incubations was also reflected by a $73-82 \%$ lower ${ }^{13} \mathrm{C}$-incorporation in $\mathrm{AOB}$ cells in the former incubations (Fig. 2, median $\mathrm{AOB}{ }^{13} \mathrm{C}$-enrichment in recirculated setup was 3.7 and 3.9 ${ }^{13} \mathrm{C}$-atom\%, in batch incubations 20.7 and $14.7{ }^{13} \mathrm{C}$-atom\% for $\mathrm{E} 1$ and $\mathrm{E} 2$, respectively). $\mathrm{AOB}$ in the flow-through incubations also showed lower ${ }^{13} \mathrm{C}$-enrichment levels $(8.2$ and 8.5 atom\% for E1 and E2, respectively) compared to batch incubations but higher enrichment than in the recirculated incubations. The lower enrichment of AOB in the recirculated compared to the flow-through incubations might be due to an accumulation of compounds leaching from the used tubing (PharMed ${ }^{\circ}$ Ismaprene, Table S1), which may negatively affect AOB. Indeed, nitrifiers have previously been reported to be sensitive to various organic compounds $[19,20]$. Use of different rubber tubing or replacing rubber tubing by glass might alleviate these effects. AOB ${ }^{13} \mathrm{C}$-enrichment was highest in batch incubations, which could be due to both the lack of stress from the continuous medium flow and the observed reaggregation of the sonicated activated sludge into larger flocs-reminiscent of native activated sludge flocs.

As expected, NOB were ${ }^{13} \mathrm{C}$-enriched in both the batch (13.3 and 4.9 atom\% for E1 and E2, respectively) and recirculated incubations (7.2 and 4.7 atom\% for $\mathrm{E} 1$ and $\mathrm{E} 2$, respectively). In contrast, as intended, the flow-through setup resulted in a substantial reduction in ${ }^{13} \mathrm{C}$-enrichment of NOB (2.0 atom\% for both E1 and E2, respectively; with consistently low ${ }^{13} \mathrm{C}$-enrichment in all NOB cells measured). This demonstrates that Flow-SIP efficiently removed the secondary substrate nitrite released by the AOB primary substrate consumers, thereby strongly limiting cross-feeding between $\mathrm{AOB}$ and NOB. The low ${ }^{13} \mathrm{C}$-enrichment of NOB in the flow-through incubations was statistically not significantly different to the ${ }^{13} \mathrm{C}$-enrichment of non-nitrifier cells (Table S2). It is unlikely that this low background ${ }^{13} \mathrm{C}$-enrichment was due to ${ }^{13} \mathrm{C}$-bicarbonate adsorption, as all samples were treated with acid before nanoSIMS analysis. It is, however, possible that at least some of the observed ${ }^{13} \mathrm{C}$-enrichment in NOB and other bacteria is due to anaplerotic reactions leading to $\mathrm{C}$-fixation by background cellular activity rather than substrate-induced autotrophic C-fixation [e.g., 21, 22]. Transfer of ${ }^{13} \mathrm{C}$-labeled metabolites from the autotrophic nitrifiers to non-nitrifier cells was negligible in all incubations, including batch and recirculated incubations (Fig. 2), which was likely due to the short incubation time $(24 \mathrm{~h})$. In contrast, other SIP studies using incubation times of several days reported significant C-isotope transfer from nitrifiers to non-nitrifiers [11, 23].

Our results demonstrate that Flow-SIP is a promising approach to significantly reduce cross-feeding in complex microbial communities and can be even successfully applied to highly aggregated samples like activated sludge flocs when they are dispersed prior to the experiment. FlowSIP and conventional SIP are complementary to each other in the analysis of such aggregated or biofilm communities. In such systems, Flow-SIP enables microbial ecologists studying microbial physiologies with drastically reduced cross-feeding, but destroys the spatial arrangement of cells, while conventional SIP retains the 3D architecture, but its results are strongly influenced by cross-feeding. We expect that Flow-SIP is ideally suited for oligotrophic fresh- or seawater samples, which predominantly harbor planktonic cells or small aggregates, and thus do not require any sonication pretreatment before incubation. Furthermore, for such samples, tracer can be directly added to sterile filtered water without the need for using artificial medium, as used for the presented proof-of-principle experiments.

Flow-SIP may, after upscaling to label more biomass, also be used in combination with DNA-, RNA- or protein- 

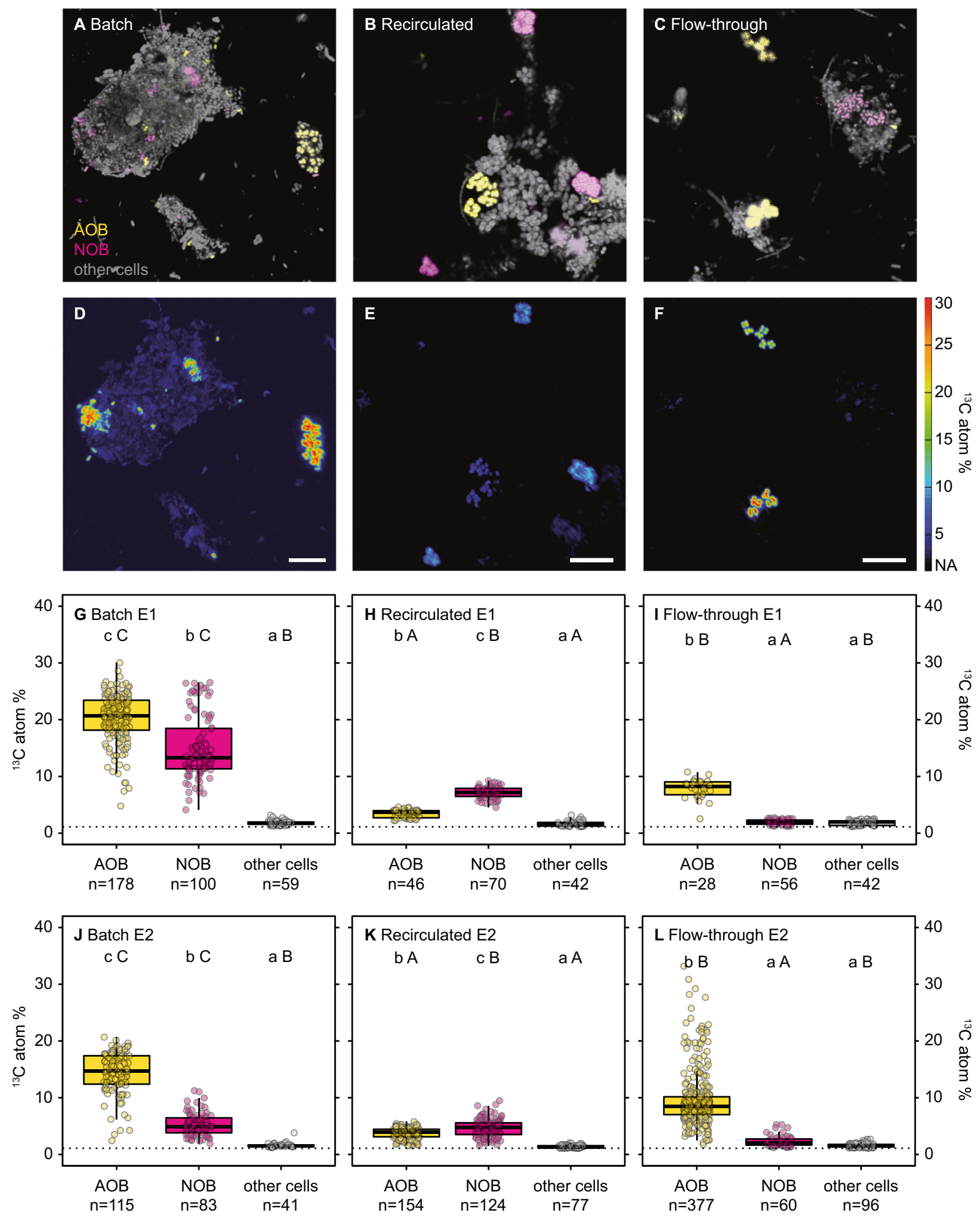

SIP, which should in comparison to conventional SIP, where cross-feeding is not inhibited, allow microbial ecologists to more precisely identify both previously known and yet unknown primary consumers of a supplied substrate.
For example, Flow-SIP with ${ }^{13} \mathrm{C}$-bicarbonate and unlabeled ammonium would allow distinguishing comammox organisms from canonical NOB, as comammox but not the canonical NOB would be active under these conditions 
Fig. 2 Single cell isotope probing of nitrifying activated sludge in batch, recirculated, and flow-through incubations. a-c Show representative FISH images of E2 (AOB in yellow; NOB in magenta; other cells counterstained by DAPI in gray) of batch, recirculated, and flow-through incubations, respectively, and $\mathbf{d}-\mathbf{f}$ show the corresponding nanoSIMS images. Scale bar is $10 \mu \mathrm{m}$ in all images. g-l Show ${ }^{13} \mathrm{C}$ labeling of $\mathrm{AOB}, \mathrm{NOB}$, and other cells quantified by nanoSIMS at the single-cell level for E1 $(\mathbf{g}-\mathbf{i})$ and E2 $(\mathbf{j}-\mathbf{l})$ in batch, recirculated, and flow-through incubations, respectively. We used FISH probe sets targeting AOB (Nitrosomonas oligotropha cluster (Cl6a192), Nitrosomonas eutropha/europea/urea cluster (NEU)) and NOB (Nitrotoga (Ntoga122), Nitrospira Lineage 1 (Ntspa1431), and Nitrospira Lineage 2 (Ntspa1151)), respectively, for differential staining of the two nitrifier groups. In $(\mathbf{g}-\mathbf{l})$, dashed lines give the ${ }^{13} \mathrm{C}$ natural abundance values of the filter surface. The number of cells analyzed per group is indicated below each boxplot. For each experiment, lower case letters indicate significant difference in ${ }^{13} \mathrm{C}$ labeling between groups (AOB, NOB, other cells) within an incubation type and upper case letters indicate significant difference between incubation types for a given group (Kruskal-Wallis test followed by Dunn's test; Statistics are given in Table S2). Boxplots depict the $25-75 \%$ quantile range, with the center line depicting the median (50\% quantile) and whiskers encompass data points within $1.5 \times$ the interquartile range.

together with the canonical ammonia oxidizers. In addition, Flow-SIP has the potential to study direct use of chemically unstable substrates by microorganisms, by distinguishing it from microbial consumption of their chemically formed decomposition products. For example, cyanate, which abiotically decays relatively fast to ammonium and carbon dioxide [24, 25], has previously been shown to serve as energy and nitrogen source for ammonia-oxidizing archaea $[25,26]$. Using Flow-SIP, cyanate could be constantly supplied, thereby strongly reducing abiotic decay. At the same time, any abiotically formed ammonium (and ammonium produced by other organisms) would be constantly removed, which should allow identifying ammoniaoxidizing microorganisms that directly use cyanate as a substrate. Furthermore, the presented approach may be coupled to fluorescence-based activity markers, where a substrate of interest and bioorthogonal noncanonical amino acids are supplied and, subsequently, translationally active cells are visualized on an epifluorescence microscope (BONCAT) [27]. In conclusion, Flow-SIP expands the toolbox of microbial ecologists interested in structure-function analyses of microbial communities and will contribute to a more precise understanding of the ecophysiology of bacteria and archaea catalyzing key processes in their natural environments.

Acknowledgements We thank Markus Schmid for help with LMD and CLSM, Stephanie Eichorst for helpful suggestions for nanoSIMS sample preparation and Marcel Kuypers for fruitful discussions and helpful comments on the manuscript. The study was supported by the ERC Advanced Grant NITRICARE (294343), the Austrian Science Fund (DK plus W 1257), DFG-Research Center/Cluster of Excellence
"The Ocean in the Earth System" at the University of Bremen (EXC 309), the University of Vienna, and the Max Planck Society.

Author contributions MW, MM, and KK designed the study. MM and KK performed experiments, with support from JLN and PN, $\mathrm{MM}$ and KK analysed the data. AS ran nanoSIMS analyses. SA modeled nitrite concentrations around ammonia oxidizer colonies. The manuscript was written by MM and KK with contributions from all coauthors.

\section{Compliance with ethical standards}

Conflict of interest The authors declare that they have no conflict of interest.

Publisher's note Springer Nature remains neutral with regard to jurisdictional claims in published maps and institutional affiliations.

Open Access This article is licensed under a Creative Commons Attribution 4.0 International License, which permits use, sharing, adaptation, distribution and reproduction in any medium or format, as long as you give appropriate credit to the original author(s) and the source, provide a link to the Creative Commons license, and indicate if changes were made. The images or other third party material in this article are included in the article's Creative Commons license, unless indicated otherwise in a credit line to the material. If material is not included in the article's Creative Commons license and your intended use is not permitted by statutory regulation or exceeds the permitted use, you will need to obtain permission directly from the copyright holder. To view a copy of this license, visit http://creativecommons. org/licenses/by/4.0/.

\section{References}

1. Boschker HTS, Nold SC, Wellsbury P, Bos D, De Graaf W, Pel R, et al. Direct linking of microbial populations to specific biogeochemical processes by ${ }^{13} \mathrm{C}$-labelling of biomarkers. Nature. 1998;392:801-4.

2. Radajewski S, Ineson P, Parekh NR, Murrell JC. Stable-isotope probing as a tool in microbial ecology. Nature. 2000;403:646-9.

3. Orphan VJ, House CH, Hinrichs K-U, McKeegan K, Delong EF. Methane-consuming archaea revealed by directly coupled isotopic and phylogenetic analysis. Science. 2001;293:484-6.

4. Manefield M, Whiteley AS, Griffiths RI, Bailey MJ. RNA stable isotope probing, a novel means of linking microbial community function to phylogeny. Appl Environ Microbiol. 2002; 68:5367-73.

5. Daebeler A, Bodelier PLE, Yan Z, Hefting MM, Jia Z, Laanbroek HJ. Interactions between Thaumarchaea, Nitrospira and methanotrophs modulate autotrophic nitrification in volcanic grassland soil. ISME J. 2014;8:1-14.

6. Gülay A, Fowler JS, Tatari K, Thamdrup B, Albrechtsen HJ, Abu Al-Soud W, et al. DNA- and RNA-SIP Reveal Nitrospira spp. as Key Drivers of Nitrification in Groundwater-Fed Biofilter. MBio. 2019;10:e01870-19.

7. Berg JS, Pjevac P, Sommer T, Buckner CRT, Philippi M, Hach $\mathrm{PF}$, et al. Dark aerobic sulfide oxidation by anoxygenic phototrophs in anoxic waters. Environ Microbiol. 2019;21:1611-26.

8. Middelburg JJ, Barranguet C, Boschker HTS, Herman PMJ, Moens T, Heip CHR. The fate of intertidal microphytobenthos carbon: An in situ ${ }^{13} \mathrm{C}$-labeling study. Limnol Oceanogr. 2000;45:1224-34. 
9. DeRito CM, Pumphrey GM, Madsen EL. Use of field-based stable isotope probing to identify adapted populations and track carbon flow through a phenol-degrading soil microbial community. Appl Environ Microbiol. 2005;71:7858-65.

10. Dumont MG, Pommerenke B, Casper P, Conrad R. DNA-, rRNAand mRNA-based stable isotope probing of aerobic methanotrophs in lake sediment. Environ Microbiol. 2011;13:1153-67.

11. Dolinšek J, Lagkouvardos I, Wanek W, Wagner M, Daims H. Interactions of nitrifying bacteria and heterotrophs: Identification of a Micavibrio-like putative predator of Nitrospira spp. Appl Environ Microbiol. 2013;79:2027-37.

12. Neufeld JD, Schäfer H, Cox MJ, Boden R, McDonald IR, Murrell JC. Stable-isotope probing implicates Methylophaga spp and novel Gammaproteobacteria in marine methanol and methylamine metabolism. ISME J. 2007;1:480-91.

13. Ho A, Angel R, Veraart AJ, Daebeler A, Jia Z, Kim SY, et al. Biotic interactions in microbial communities as modulators of biogeochemical processes: Methanotrophy as a model system. Front Microbiol. 2016;7:1-11.

14. Lueders T, Manefield M, Friedrich MW. Enhanced sensitivity of DNA- and rRNA-based stable isotope probing by fractionation and quantitative analysis of isopycnic centrifugation gradients. Environ Microbiol. 2004;6:73-78.

15. Maxfield PJ, Hornibrook ERC, Evershed RP. Estimating highaffinity methanotrophic bacterial biomass, growth, and turnover in soil by phospholipid fatty acid ${ }^{13} \mathrm{C}$ labeling. Appl Environ Microbiol. 2006;72:3901-7.

16. Pan C, Fischer CR, Hyatt D, Bowen BP, Hettich RL, Banfield JF. Quantitative tracking of isotope flows in proteomes of microbial communities. Mol Cell Proteom. 2011;10:1-11.

17. Albertsen M, Hansen LBS, Saunders AM, Nielsen PH, Nielsen KL. A metagenome of a full-scale microbial community carrying out enhanced biological phosphorus removal. ISME J. 2012;6:1094-106.
18. Munck C, Albertsen M, Telke A, Ellabaan M, Nielsen PH, Sommer MOA. Limited dissemination of the wastewater treatment plant core resistome. Nat Commun. 2015;6:8452.

19. Krümmel A, Harms H. Effect of organic matter on growth and cell yield of ammonia-oxidizing bacteria. Arch Microbiol. 1982;133:50-54.

20. Spieck E, Lipski A. Cultivation, growth physiology, and chemotaxonomy of nitrite-oxidizing bacteria. In: Klotz MG, editor. Methods in enzymology, 1st ed. San Diego, USA: Elsevier Inc.; 2011. pp 109-30.

21. Li W. Estimating heterotrophic bacterial productivity by inorganic radiocarbon uptake: importance of establishing time courses of uptake. Mar Ecol Prog Ser. 1982;8:167-72.

22. Roslev P, Larsen MB, Jørgensen D, Hesselsoe M. Use of heterotrophic $\mathrm{CO}_{2}$ assimilation as a measure of metabolic activity in planktonic and sessile bacteria. J Microbiol Methods. 2004;59:381-93.

23. Okabe S, Kindaichi T, Ito T. Fate of ${ }^{14} \mathrm{C}$-labeled microbial products derived from nitrifying bacteria in autotrophic nitrifying biofilms. Appl Environ Microbiol. 2005;71:3987-94.

24. Dirnhuber P, Schütz F. The isomeric transformation of urea into ammonium cyanate in aqueous solutions. Biochem $\mathrm{J}$. 1948;42:628-32.

25. Palatinszky M, Herbold C, Jehmlich N, Pogoda M, Han P, Von BergenM, et al. Cyanate as an energy source for nitrifiers. Nature. 2015;524:105-8.

26. Kitzinger K, Padilla CC, Marchant HK, Hach PF, Herbold CW, Kidane AT, et al. Cyanate and urea are substrates for nitrification by Thaumarchaeota in the marine environment. Nat Microbiol. 2019;4:234-43.

27. Hatzenpichler R, Scheller S, Tavormina PL, Babin BM, Tirrell DA, Orphan VJ. In situ visualization of newly synthesized proteins in environmental microbes using amino acid tagging and click chemistry. Environ Microbiol. 2014;16:2568-90. 